\title{
Nanoscale Polarization of the Vaccinia Virus Entry Fusion Complex Drives Efficient Fusion
}

Authors: Robert D. M. Gray ${ }^{\dagger, 1,2}$, David Albrecht ${ }^{\dagger}, 1$, Corina Beerli ${ }^{1}$, Moona Huttunen ${ }^{1}$, Gary H. Cohen $^{3}$, Ian J. White ${ }^{1}$, Jemima J. Burden ${ }^{1}$, Ricardo Henriques* ${ }^{1,4}$, Jason Mercer*1

\section{Affiliations:}

${ }^{1}$ MRC Laboratory for Molecular Cell Biology. University College London, Gower Street, London, WC1E 6BT, United Kingdom

${ }^{2}$ Centre for Mathematics and Physics in Life Sciences and Experimental Biology (CoMPLEX), University College London, Gower Street, London, WC1E 6BT, United Kingdom

${ }^{3}$ Department of Microbiology, School of Dental Medicine, University of Pennsylvania, Philadelphia, PA

${ }^{4}$ Department of Cell and Developmental Biology, University College London, Gower Street, London, WC1E 6BT, United Kingdom

*Correspondence to: r.henriques@ucl.ac.uk (R.H.); jason.mercer@ucl.ac.uk (J.M.)

$\dagger$ These authors contributed equally to this work.

bioRxiv deposit: https://doi.org//0.1 101/360073 
Introductory paragraph:

To achieve efficient binding and subsequent fusion most enveloped viruses encode between 1 and 5 proteins ${ }^{1}$. For many viruses, the clustering of fusion proteins, and their distribution on virus particles, is crucial for fusion activity ${ }^{2,3}$. Poxviruses, the most complex mammalian 
The Poxviridae, including the causative agent of smallpox (variola), monkeypox, and the smallpox vaccine (vaccinia virus) encode 4 proteins for binding and 11 proteins for fusion ${ }^{4}$ The binding proteins (D8, H3, A26 and A27) are not individually essential ${ }^{6-9}$. However, genetic repression of any of the 11 proteins required for fusion, collectively termed the entry fusion complex $(\mathrm{EFC})^{5}$, results in formation of morphologically normal virions incompetent for hemifusion (A16, A21, F9, G3, G9, H2, J5 and O3) or full fusion (A28, L1 and L5) ${ }^{5}$. All EFC components are transmembrane proteins and 9 (A16, A21, A28, G3, G9, H2, J5, L5 and O3) form a stable core complex with which L1 and F9 associate ${ }^{5}$.

EFCs within MVs are required for fusion of both infectious forms of Poxviridae ${ }^{10}$ : singlemembrane mature virions (MVs), and double-membrane extracellular enveloped virions (EEVs), which shed their outermost membrane allowing for fusion of the underlying MV-like particle $^{11,12,13}$. Here we used a combination of electron microscopy (EM), super-resolution microscopy, single-particle analysis and a large collection of virus mutants to investigate virion binding and fusion orientation, the spatial distribution of binding and fusion proteins on individual vaccinia virus (VACV) particles, and how fusion protein distribution correlates with fusion activity.

Noting an orientation bias in poxvirus EM studies (Supplementary Table 1), we analysed the orientation of VACV MV binding and plasma membrane (PM) fusion using scanning- and transmission- EM, respectively. Quantification of binding orientation showed that $>99 \%$ of MVs 
bound to the cell surface on their sides (Fig. 1a). When fusion was forced by lowering the $\mathrm{pH}^{13-}$

${ }^{15}$, fusion of virion and cell membranes occurred at MV tips in $96 \%$ of cases (Fig. 1b). These results were consistent with the literature in which $98 \%$ of MV binding events occurred at virion sides, and 100\% of fusion events at tips (Supplementary Table 1).

These results suggested that viral membrane proteins may be organized into functional domains. To investigate this we applied structured illumination microscopy (SIM) ${ }^{16}$ and single-particle averaging to generate models of the distribution of binding and EFC proteins in MVs ${ }^{17,18}$. For this, mCherry-tagged core protein A4 was used to identify virion position and orientation (Fig. 1c), and EGFP-tagged protein $\mathrm{A} 13^{19}$ as a viral membrane marker (Fig. 1c). VACV binding (A27 \& D8) and EFC proteins (A21, A28, F9, J5, H2 and L1) were visualized by immunofluorescence. The localization models showed that MV binding proteins reside at virion sides and EFC components localize to virion tips, independent of virion orientation (Fig. 1c, Supplementary Fig. 1). Polarity factor quantification indicated that binding proteins were enriched 1.2-fold at the sides, and EFC proteins 1.7-fold at the tips of virions (Fig. 1d, Supplementary Fig. 2).

The EFC is held together by a complex network of interactions ${ }^{20,21}$. Repression of any core component results in disruption of the EFC into sub-complexes without compromising the expression or virion incorporation of other EFC proteins ${ }^{5}$. This allowed us to investigate whether the polarized distribution of binding and EFC components depends upon EFC intactness. MVs lacking EFC core components A28, G9 or O3 were immunolabelled for binding protein D8 and EFC protein L1. Localization models showed that D8 distribution was unaffected by loss of EFC 
components, while L1 was redistributed evenly around the MV membrane (Fig. 1e). Models of A21, A28, F9, H2 and J5 on A28(-), G9(-) and O3(-) MVs showed that all EFC components were depolarized in these mutants (Supplementary Fig. 3a). No redistribution of D8 or EFC proteins was seen on virions lacking the binding protein H3 (Fig. 1e, Supplementary Fig. 3a, b). Polarity factor quantification confirmed that deletion of EFC core components does not alter D8 localization, while shifting EFC distribution from polarized to isotropic (Fig. 1f, Supplementary Fig. 3b). Collectively these results show that VACV binding and fusion machineries are organized as distinct functional domains within the viral membrane (Fig. 1g), and that the polarized distribution of EFCs relies on their intactness.

Using stochastic optical reconstruction microscopy (STORM) ${ }^{22}$, we extended our investigation to single virions. Immunolabeling of D8 and L1 was performed on WT, EFC [A28(-), G9(-) and O3(-)], and binding protein $(\Delta \mathrm{H} 3)$ mutant MVs. As expected, D8 was distributed to the sides of all virions, while L1 was polarized to the tips of WT and $\Delta \mathrm{H} 3 \mathrm{MVs}$ and distributed throughout the membrane in A28(-), G9(-) and O3(-) virions (Fig. 2a, Supplementary Fig. 4a). STORM revealed that D8 and L1 were localized to distinct clusters in WT and $\Delta \mathrm{H} 3 \mathrm{MVs}$ (Fig. 2a). D8 clusters appeared unaffected in EFC mutants, while L1 clusters were largely disrupted (Fig. 2a). Similar D8 and L1 distributions were observed on WT MVs stained with fluorescently conjugated primary antibodies or Fab fragments, indicating that clustering was not induced by signal amplification through secondary antibodies (Supplementary Fig. 4b, c) ${ }^{23}$. Additionally, STORM showed that both D8 distribution and L1 polarization is maintained on the MV-like particles within EEVs (Supplementary Fig. 5). 
To analyse D8 and L1 clustering on MVs we applied Voronoi tessellation (SR-Tesseler

software $^{24}$ ). Labelling of A13-EGFP with fluorescently conjugated anti-EGFP nanobodies was used to determine a baseline for clustering (Supplementary Fig. 4d). Images were subjected to localization, segmentation and cluster identification by SR-Tesseler (Supplementary Fig. 6a). The clustering threshold was set for each particle at 3-fold the average density (Supplementary Fig. 6b). This analysis was used to quantify D8 and L1 clustering in WT, EFC [A28(-), G9(-), O3(-)], and binding ( $\Delta \mathrm{H} 3)$ mutants (Fig. 2b). The percent of D8 localizations in clusters, and the number of D8 clusters, did not differ between WT and EFC- or binding- mutants (Fig. 2c, d). Conversely, L1 localizations in clusters was reduced from $44 \%$ to $3 \%$, and L1 clusters from 8 to $\leq 4$, on WT vs. EFC mutants (Fig. 2e,f, Supplementary Fig. 6c,d). The total cluster area of L1 was reduced from $4,400 \mathrm{~nm}^{2} /$ virion on $\mathrm{WT}$ and $\Delta \mathrm{H} 3 \mathrm{MVs}$, to below the imaging resolution on A28(-), G9(-) and O3(-) virions (Supplementary Fig. 6e). The loss of fusion machinery polarization and clustering observed in EFC mutants correlated with the fusion defects exhibited by these viruses ${ }^{5,25}$.

It was reported by Laliberte et al. that unlike other EFC mutants, A28(-) MVs are capable of directing hemifusion ${ }^{25}$. That EFC polarization and clustering were equally disrupted in A28(-) and other EFC mutants, suggested that polarization and clustering may be more important for full fusion than hemifusion. To test this, a viral protein that is not a component of the EFC, whose loss affects MV fusion, was needed. The VACV protein A27 has multiple roles in the virus lifecycle including virus binding, fusion and the intracellular transport and wrapping of $\mathrm{MVs}^{9,26-30}$. Direct evidence of A27's involvement in fusion comes from Vazquez and Esteban, who showed that A27(-) virions cannot mediate acid-induced cell-cell fusion ${ }^{26}$. This phenotype 
was confounded by the fact that the A27(-) virus had no defect in MV production. 24h-yield and cell-cell fusion experiments confirmed that A27(-) MVs display no defect in MV production (Fig. 3a), but are 8-fold less capable of mediating cell-cell fusion than A27(+) virions (Fig. 3b, c). 
Thus we asked if A27(-) MVs can undergo acid-induced hemi- and full- fusion with cells ${ }^{25,32}$ using the assay illustrated in Figure $4 a^{32}$. MVs containing an EGFP core were labelled with the self-quenching membrane dye R18. The MVs were bound to cells in medium at $4{ }^{\circ} \mathrm{C} / \mathrm{pH} 7.4$, and $37^{\circ} \mathrm{C} / \mathrm{pH} 5.0$ medium was added to induce MV fusion with the plasma membrane. Proton influx into virions quenches the $\mathrm{pH}$-sensitive EGFP core fluorescence, followed by R18 dequenching as a result of lipid mixing during hemifusion ${ }^{32}$. Upon full fusion, cores are exposed to cytosolic $\mathrm{pH}$ allowing for recovery of EGFP core fluorescence. Measurement of the R18 and EGFP fluorescence over time allows for quantitative assessment of MV hemi- and full- fusion ${ }^{25,32}$. Comparison of R18 dequenching rates indicated that $\mathrm{A} 27(+)$ and $\mathrm{A} 27(-) \mathrm{MV}$ hemifusion was comparable (Fig. 4b). While core EGFP quenching occurred in both A27(+) and A27(-) MVs, only A27(+) MVs displayed EGFP recovery (Fig. 4c). These results indicated that A27(-) MVs can undergo hemifusion but are impaired in their ability to mediate full fusion.

To assess how A27(-) MVs produce equivalent numbers of infectious virus as A27(+) MVs despite being impaired for full fusion, cells infected with A27(+) or (-) MVs expressing EGFP under the control of an early viral promoter were monitored from 2-8 hours post infection (hpi) (Fig. 4d, Supplementary Fig. 9). This assay, employed as a surrogate to assess delayed VACV fusion $^{25,32}$, showed detectable early gene expression in $61 \%$ of $\mathrm{A} 27(+)$ infected cells, as opposed to $38 \%$ of $\mathrm{A} 27(-)$ infected cells at $2 \mathrm{hpi}$. The percentage of A27(+) infected cells expressing early genes climbed only slightly over 8h, while the percentage of A27(-) infected cells expressing early genes climbed steadily, reaching the levels of A27(+) within 8h. The ability of A27(-) MVs to overcome delayed full fusion kinetics (Fig. 4c) is consistent with the production of equivalent numbers of MVs in A27(+) and A27(-) infections over a 24h period (Fig. 3a). 
The experimental data indicates that clustered polarization of the fusion machinery is important for VACV full fusion efficiency. To further explore the impact of EFC clustering on fusion we implemented a simple kinetic model ${ }^{33}$. The contact area between the virus and cell was modelled to contain a variable density of fusion complexes which stochastically activate to drive fast hemifusion followed by rate-limiting full fusion. By varying fusion complex density within the virus-cell contact area, we could assess how fusion complex clustering effects the rate of full fusion. We tested this model at two fusion thresholds ${ }^{33}$ : low, which requires 3 activated fusion complexes and high, which requires 5. Simulations of 1,000 viruses indicated that regardless of the fusion threshold, full fusion kinetics slow down when fusion complex density is reduced from high to low (Fig. 4e). Notably, at the high threshold the rate of fusion was more dependent on complex density, indicating that full fusion is highly dependent on complex clustering.

Based on these collective findings, we propose that the organization of poxvirus membrane proteins into functional domains is important for virus entry, and that EFC polarization and clustering is critical for efficient MV and EEV virus-cell fusion. The only other documented example of polarized virus fusion machinery is on $\mathrm{HIV}-1^{34}$. The authors propose that Env clustering is important due to the low number of Env trimers present in the viral membrane ${ }^{34}$. Formation of a single Env cluster on mature virions is required for efficient CD4 engagement and fusion at the PM. While a minority of MVs and EEVs enter by PM fusion, the majority of VACV enter by acid-mediated endocytosis ${ }^{13,15,35-39}$. This, together with our findings, invokes a model whereby VACV MVs bind to the PM in a side-on orientation (see Fig. 4f). This is 
consistent with MV binding data, the position of the binding machinery on the virus and accounts for the low number of direct PM fusion events observed after low pH-treatment.

Upon endocytosis - and in the case of EEVs, low pH-mediated rupture of the outer membrane ${ }^{32}-$ 201 both MVs and MV-like particles are completely enveloped within cellular membrane 202 compartments. This allows for virus orientation-independent contact between virion tips and the 203 limiting membrane of endosomes in order to drive EFC-mediated fusion and core deposition. Consistent with this model, MV and EEV fusion from endosomes has only been observed to 205 occur at virion tips ${ }^{15,40,41}$.

In sum, by revealing the nanoscale organization of the poxvirus membrane and showing the consequences of its disruption, we demonstrate that virion protein architecture is critical to virus productive infection. 
Methods:

\section{Cells and viruses}

African green monkey kidney (BSC-40) cells and human HeLa cells were cultivated in Dulbecco's modified Eagle's medium (DMEM) supplemented with 10\% heat-inactivated fetal bovine serum (FBS), $2 \mathrm{mM}$ Glutamax, 100 units $/ \mathrm{mL}$ penicillin and $100 \mu \mathrm{g} / \mathrm{mL}$ streptomycin, $100 \mu \mathrm{M}$ nonessential amino acids and $1 \mathrm{mM}$ sodium pyruvate. Routine mycoplasma tests of the cell culture medium were negative. Recombinant VACV were based on VACV strain Western Reserve (WR). WR mCherry-A4 and WR L4-mCherry/EGFP-F17 were described previously as WR mCherry-A513 and WR EGFP-F17 VP8-mCherry42. WR mCherry-A4 F13-EGFP was described previously as WR mCherry-A5 F13-GFP13. $\Delta \mathrm{H} 3, \mathrm{~A} 28(+/-), \mathrm{G} 9(+/-)$ and $\mathrm{O} 3(+/-)$ were described previously as vH3 $\Delta 43$, vA28-HAi44, vG9i45 and vO3-HAi46 respectively. WRA27(+/-) was previously described as VVIndA27L30. WR A4-mCherry/A13-EGFP, WRA27(+/-) EGFP-A4 and WRA27(+/-) E/L EGFP were constructed as previously described42. For WR A4-mCherry/A13-EGFP, A13 was replaced with A13-EGFP in its endogenous locus within WR A4-mCherry virus. First, primers gcgectcgagatctcgacattgttgaatcattattac and gegcggtacccaccagaagtattttggagcc were used to amplify the A13 region from the viral genome with XhoI and KpnI sites, which was inserted in the pBluescript II KS backbone. Then an EGFP tag was inserted with Gibson assembly. To generate WRA27(+/-) EGFP-A4, A4 was replaced with EGFP-A4 in its endogenous locus within WRA27(+/-). First, primers ccatcgatgatgactataggacaagaaccetcctc and cggaattccgcttgaacagcattgc were used to amplify the A4 region from the viral genome with EcoRI and ClaI sites, which was then inserted into the pBluescript II KS backbone. Then, an EGFP tag was inserted using Gibson assembly. For WRA27(+/-) E/L EGFP, EGFP under the control of an early/late viral promoter was inserted 
between the H2-H3 loci of the WRA27(+/-) virus. First, primers gcgcggtacctagcegctggtaaggatga and gcgcggtaccgcagatactggataatgccg were used to amplify the H2-H3 region from the viral genome with KpnI sites, which was then inserted into the pUC/neo backbone. Then, E/L EGFP was introduced into the H2-H3 locus using primers gtacaagtaagaattctgttagataaatgcggtaacgaat and tttagtaatatggaatagaagcttaaaaattgaaatttt to introduce EcoRI and HindIII sites into the $\mathrm{H} 2-\mathrm{H} 3$ region and the primers aagcttaaaaattgaaattttatttttttt and gaattcttacttgtacagctcgtcc to introduce the same sites into E/L EGFP. Briefly, BSC-40 cells were infected the parental viruses and subsequently transfected with linearized plasmid containing the region of genome to be replaced, flanked 5' and 3' by 300bp of genomic sequence for targeted homologous recombination. Recombinant viruses were selected by fluorescence through 4 rounds of plaque purification. All viruses were produced in BSC-40 cells, and MVs purified from cytoplasmic lysates by banded on sucrose gradients, as previously described ${ }^{35}$. For WRA27(+/-) and its derivatives, virus stocks were generated in the presence or absence of $2 \mathrm{mM}$ Isopropyl $\beta$-D-thiogalactoside (IPTG, Sigma) to obtain A27+ or A27- MVs. WRA28(+), WRG9(+) and WRO3(+) stocks were produced with $100 \mu \mathrm{M}, 50 \mu \mathrm{M}$ and $20 \mu \mathrm{M}$ IPTG respectively. EEVs were prepared as previously described ${ }^{13}$. Briefly, RK13 cells were infected with WR F13-EGFP mCherry-A4 at MOI 1 and EEVs purified from the supernatant at 24hpi. Debris was removed by centrifugation at $1,000 \mathrm{xg}$, and EEVs concentrated by centrifugation at $38,000 \mathrm{x} \mathrm{g}$ before being resuspended in $1 \mathrm{mM}$ Tris $\mathrm{pH} 9$.

\section{Antibodies}

Anti-L1 mouse monoclonal antibody (clone 7D11) was purified from a hybridoma cell line kindly provided by Bernard Moss (National Institutes of Health, Bethesda, MD) with permission of Alan Schmaljohn (University of Maryland, Baltimore, MD). Anti-D8 rabbit polyclonal 
antibody was made by immunizing a rabbit with purified D8 protein and adjuvant. Anti-A17 rabbit polyclonal antibody was a kind gift from Jacomine Krijnse-Locker. Antibodies against viral proteins A21 (R206), A28 (R204), F9 (R192), H2 (R202), J5 (R264), were produced by GHC using purified recombinant baculovirus-expressed proteins as previously described ${ }^{47}$.

\section{Structured illumination imaging}

High performance coverslips $(18 \times 18 \mathrm{~mm} 1.5 \mathrm{H}$, Zeiss $)$ were washed as previously described ${ }^{17}$. Purified virus was diluted in $1 \mathrm{mM}$ Tris $\mathrm{pH}$, bound to the ultra-clean coverslips for 30 min and fixed with 4\% Formaldehyde. Samples were washed 3 times with PBS prior to mounting for imaging. To visualize membrane proteins, virus was blocked after fixation using 5\% bovine serum albumen (BSA, Sigma) in PBS for $30 \mathrm{~min}$, incubated in 1\% BSA in PBS with primary antibody, followed by Alexa Fluor 488-conjugated goat anti-mouse or anti-rabbit secondary antibody for 1 hour each. Samples were washed 3 times with PBS after each staining step. The coverslips were mounted in Vecta Shield (Vector Labs) and sealed with nail polish.

SIM imaging was performed using Plan-Apochromat $63 \times / 1.4$ oil DIC M27 objective, in an Elyra PS.1 microscope (Zeiss). Images were acquired using 5 phase shifts and 3 grid rotations, with the $561 \mathrm{~nm}(32 \mu \mathrm{m}$ grating period) and the $488 \mathrm{~nm}$ (32 $\mu \mathrm{m}$ grating period) lasers, and filter set 3 (1850-553, Zeiss). 2D images were acquired using a sCMOS camera and processed using the ZEN software (2012, version 11.0.3.190, Zeiss). For channel alignment, TetraSpeck beads (ThermoFisher) were mounted on a slide, imaged using the same image acquisition settings and used for the alignment of the different channels.

\section{Single-particle analysis}

Individual viral particles were extracted from the SIM images. Seed images were generated with VirusMapper as described previously ${ }^{17}$. VirusMapper models were then created by registration 
of the entire set of particles according to cross-correlation with the seeds and calculation of a 284 weighted average of a subset of particles. These values of (n) are shown in Supplementary Figs. 2853 and 7. Models are normalized and intensity therefore does not reflect protein abundance.

\section{$286 \quad$ Polarity factor}

287 Polarity factors were calculated directly from the sets of particles used to generate the models 288 (Supplementary Fig. 2). Following the cross-validation method of Szymborska et al. ${ }^{48}$, particles 289 were randomly divided into subsets of 50 particles and separately averaged. Radial profiles were 290 generated from these images by transforming from $x-y$ coordinates to $r-\theta$. The radial profiles 291 were divided into four regions according to a parameter $\varphi$ and the mean intensity within the viral 292 membrane in these regions was evaluated. The four regions were defined in $\theta$ by:

$$
\begin{gathered}
I_{1}: 360^{\circ}-\varphi<\theta \text { or } 0<\theta<\varphi \\
I_{2}: \varphi<\theta<180^{\circ}-\varphi \\
I_{3}: 180^{\circ}-\varphi<\theta<180^{\circ}+\varphi \\
I_{4}: 180^{\circ}+\varphi<\theta<360^{\circ}-\varphi
\end{gathered}
$$
image that intersect at angle $\varphi$ and divide the image symmetrically about the horizontal and vertical axes. The polarity factor was then calculated as

$$
p=\frac{I_{2}+I_{4}}{I_{1}+I_{3}}
$$

A value for $\varphi$ was used that resulted in a mean polarity factor of 1 for the A4 core protein $\left(48.5^{\circ}\right)$. Values quoted in the text were calculated by averaging the mean polarity factors for the sets of binding and fusion proteins.

\section{Labelling of primary antibodies}


Mouse monoclonal antibody (IgG) against L1 from hybridoma supernatant and rabbit polyclonal antibody against D8 from whole serum were purified with a Nab Protein A/G Spin Kit (Thermo Scientific) according to the manufacturer's instructions. Fab fragments were generated from primary antibodies with a Pierce Fab Preparation Kit (Thermo Scientific). Antibodies and Fab fragments were buffer exchanged into $0.1 \mathrm{M} \mathrm{NaHCO}_{3} \mathrm{pH} 8.2$ and concentrated to $>1 \mathrm{mg} / \mathrm{ml}$ with Amicon Ultra centrifugal filters $10 \mathrm{kDa}$ MWCO (Merck). Antibodies and Fab fragments were custom-labelled with a 50-fold molar excess with AlexaFluor647-NHS (Invitrogen) for 1h at RT. The reaction was quenched by addition of $2 \mu 11 \mathrm{M}$ Tris $\mathrm{pH} 9$. Unreacted dye was removed by three passes through Zeba-Spin columns 3.5K MWCO (Thermo Scientific).

\section{STORM imaging}

Single-molecule localisation microscopy was performed by direct stochastic optical reconstruction microscopy $(\mathrm{dSTORM})^{49}$, a method developed based on STORM $^{22}$. High performance coverslips $(18 \mathrm{~mm}, 1.5 \mathrm{H}$, Zeiss) were washed in ultrapure ethanol (Sigma) and deionized water to clean them and make their surface hydrophobic. Purified MVs or EEVs were diluted in $20 \mu \mathrm{l} 1 \mathrm{mM}$ Tris $\mathrm{pH}$ 9, placed in the centre of the clean coverslips for $30 \mathrm{~min}$ and bound virus fixed with 4\% EM-grade formaldehyde (EMS). Virus was blocked after fixation using 5\% BSA (Sigma), 1\% FCS, 0.2-1\% TritonX-100 in PBS for $30 \mathrm{~min}$. Virus was immunostained in $5 \%$ BSA in PBS with primary antibodies at $4{ }^{\circ} \mathrm{C}$ overnight and Alexa Fluor 647-conjugated secondary antibodies (Invitrogen) for 1-2 hours at RT. Samples were washed 3 times with PBS after each staining step. Optionally, a post-fixation step with 4\% PFA was included. Autofluorescence was quenched by brief incubation in $0.25 \%(\mathrm{w} / \mathrm{v}) \mathrm{NH}_{4} \mathrm{Cl}$ in $\mathrm{PBS}$. Coverslips were mounted on a Secure-Seal incubation chamber (EMS) in BME-buffer [1\% (v/v) $\beta$-mercaptoethanol (Sigma), $150 \mathrm{mM}$ Tris, 1\% glucose, 1\% glycerol, $10 \mathrm{mM} \mathrm{NaCl}, \mathrm{pH} 8]$ or in 
MEA-buffer [50 mM cysteamine (Sigma), 3\% (v/v) OxyFluor (Oxyrase Inc), 20\% sodium $324 \quad$ lactate (Sigma), $\left.\mathrm{PBS}, \mathrm{pH} 8^{50}\right]$.

Imaging was performed on an Elyra PS.1 inverted microscope (Zeiss) using an alpha PlanApochromat $100 \times / 1.46$ NA oil DIC M27 objective with a 1.6x tube lense and an iXon 897 EMCCD camera (Andor). Images were acquired at 25-30ms exposure time with $642 \mathrm{~nm}$ excitation at $100 \%$ laser power and a $655 \mathrm{~nm}$ LP filter. Fluorophore activation was dynamically controlled with a $405 \mathrm{~nm}$ laser at $0-2 \%$ laser power. Images were processed in Fiji ${ }^{51}$ using ThunderSTORM ${ }^{52}$. Localizations were fitted with a maximum-likelihood estimator, lateral drift corrected by cross-correlation, localizations $<20 \mathrm{~nm}$ apart within $\leq 1$ frames merged, and images rendered using a Gaussian profile with the NanoJ-Orange LUT (NanoJ). Lateral resolution was $25 \mathrm{~nm}$, determined by FRC. Dual-colour STORM and SIM images (Supplementary Fig. 4, 5) were registered using $\mathrm{NanoJ}^{53}$.

\section{SR-Tesseler analysis}

Cluster analysis was performed with SR-Tesseler ${ }^{24}$. Localization tables from ThunderSTORM were imported and Voronoi diagrams created. Individual virions were selected as regions of interest and segmented as single objects with a density factor $\delta$ of $0.1-0.5$. Within individual objects, clusters were identified with $\delta=3$ which yielded $<2 \%$ clustering in the non-clustered reference probe A13-EGFP (Supplementary Fig. 6). Statistical analysis was performed in GraphPad Prism (Prism Software). Significance (unpaired t-test): $* \mathrm{p}<0.05 ; * * \mathrm{p}<0.01 ; * * * \mathrm{p}$ $<0.001$.

\section{Ripley’s H-function analysis}

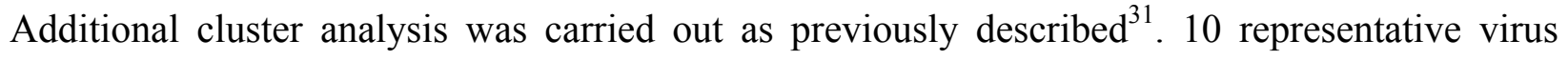
particles were selected from STORM images of L1 on WT, A27(+/-), G9(-) VACV 
(Supplementary Fig. 8e). Localizations within the selected ROIs were separately used to calculate the Ripley's H-function as a function of increasing radius $H(r)$ according to:

$$
K(r)=\frac{A}{n(n-1)} \sum_{j}^{n} \sum_{i}^{n} \delta_{i j}(r)
$$

where $A$ is the approximate area of the particle, $n$ is the number of localizations and $\delta_{i j}(r)=1$ if $\left|\boldsymbol{x}_{i}-\boldsymbol{x}_{j}\right|<r ; 0$ otherwise

where $\boldsymbol{x}_{i}$ is the spatial location of localization $i$. Then

$$
L(r)=\frac{1}{\pi} \sqrt{K(r)}
$$

and

$$
H(r)=L(r)-r
$$

so that the expected value of $H(r)$ is 0 for a random uniform distribution, and values of $H(r)$ above 0 indicate clustering at a range of approximately $r$. Custom Python scripts were used to calculate the H-function from STORM localization tables. The mean of $H(r)$ and the $95 \%$ confidence interval were evaluated for each case.

\section{4-hour yields}

Confluent BSC-40 cells were infected with A27(+/-) virus in DMEM at MOI 1. After 60 minutes the inoculum was removed and DMEM with serum and 2mM IPTG was added for 24 hours. Cells were harvested, resuspended in $100 \mu 1 \mathrm{mM}$ Tris pH 9 and freeze-thawed 3 times in liquid nitrogen. The virus concentration was then measured by plaque assay.

\section{Fusion from without}

WRA27(+/-) was bound to HeLa cells grown on coverslips on ice at MOI 10 for 1 hour. Cells were washed and incubated at $37^{\circ} \mathrm{C}$ with $20 \mathrm{mM}$ MES pH 5 or 7.4 for 5 mins, then washed and incubated in full medium for 2 hours. Cells were fixed with 4\% formaldehyde in PBS for 20 
mins, blocked and permeabilized using $0.1 \%$ Triton X-100 and 5\% BSA in PBS, stained with Alexa Fluor 488 phalloidin and Hoechst 33258 (both Invitrogen) for in PBS for 1 hour. Samples were then mounted on slides with ProLong Gold Antifade Mountant (Thermo Fisher). Samples were imaged on a Leica TCS SP8 STED 3x microscope in confocal mode running LAS X (Version 2.01) acquisition software. Alexa Fluor 488 fluorescence was excited using the $488 \mathrm{~nm}$ line and Hoechst was excited with the 405nm LED. Z-stacks were acquired at a scan speed of $400 \mathrm{~Hz}$ in bidirectional scan mode using a Hybrid Detector (HyD, Standard mode) and an Acousto-Optical Beam Splitter for filtering and time-gating of 0.5-8ns. The fusion index was calculated from maximum intensity projections of the stacks as described previously ${ }^{54}$.

\section{Bulk fusion measurements}

MVs were labelled by incubating with $22.5 \mu \mathrm{M}$ R18 (ThermoFisher) in $1 \mathrm{mM}$ Tris $\mathrm{pH} 9$ at room temperature for 2 hours. Labelled viruses were pelleted by centrifugation at 16,000g for $10 \mathrm{~min}$ at $4{ }^{\circ} \mathrm{C}$ and resuspended in $1 \mathrm{mM}$ Tris $\mathrm{pH} 9$ twice to remove excess $\mathrm{R} 18$. Labelled viruses were bound to $7 \times 10^{5}$ HeLa cells at MOI 30 in DMEM on ice for 1 hour. Cells were sedimented by centrifugation at $300 \mathrm{~g}$ for $5 \mathrm{mins}$ at $4^{\circ} \mathrm{C}$ then resuspended in $100 \mu \mathrm{l}$ PBS. The cell suspension with bound virions was added to $630 \mu 1$ prewarmed PBS in a quartz cuvette. After 2 min, the $\mathrm{pH}$ in the cuvette was lowered by addition of $100 \mu 1100 \mathrm{mM}$ MES resulting in a $\mathrm{pH}$ of 5.0. After the acquisition all R18 was dequenched by the addition of $83 \mu 110 \%$ Triton X-100 in PBS. R18 fluorescence was normalized to the signal intensity after Triton X-100 addition. R18 fluorescence was measured using a Horiba FluoroMax 4 (Horiba Jobin Yvon) spectrofluorometer with an excitation wavelength of $560 \pm 5 \mathrm{~nm}$ and an emission wavelength of $590 \pm 5 \mathrm{~nm}$. To measure EGFP fluorescence, unlabelled viruses were bound to cells and the $\mathrm{pH}$ 
was lowered as above, and fluorescence was recorded using an excitation wavelength of $488 \pm 5$ $\mathrm{nm}$ and an emission wavelength of $509 \pm 10 \mathrm{~nm}$.

\section{Flow cytometry}

HeLa cells were infected with WR A27(+/-) E/L EGFP at MOI 4 in DMEM and full medium containing $10 \mu \mathrm{M}$ AraC (Sigma) was added after 1 hour. At the indicated times cells were washed with PBS, trypsinized and resuspended in PBS and fixed with 4\% formaldehyde in PBS. Cells were then sedimented by centrifugation at $300 \mathrm{~g}$ for $5 \mathrm{mins}$ and resuspended in PBS with 2\% FBS and 5mM EDTA. Flow cytometry was performed with a Guava EasyCyte HT flow cytometer, recording the EGFP fluorescence with the $488 \mathrm{~nm}$ laser. Analysis of the flow cytometry data was performed with the FlowJo software package.

\section{Fusion kinetics mathematical model}

We modelled the process of fusion undergone by a single fusion complex as a fast irreversible hemifusion step followed by a rate-limiting, irreversible full fusion step.

$$
I \stackrel{k_{h f}}{\longrightarrow} H F \stackrel{k_{\text {fuse }}}{\longrightarrow} F
$$

Following similar work on influenza ${ }^{33}$, for each virus we required that $T_{f}$ (fusion threshold) fusion complexes transition to state $F$ before the virus is considered fused. We simulated the contact area with the cell as containing an initial density $\rho_{0}$ (high density), $\frac{\rho_{0}}{3}$ (medium density) and $\frac{\rho_{0}}{10}$ (low density) of fusion complexes. We then modelled the effects of fusion complex density on fusion kinetics at two different values of $T_{f}$ : low $\left(T_{f}=3\right)$ and high $\left(T_{f}=5\right)$. The true value of $T_{f}$ is unknown for vaccinia but for influenza is thought to be in this range.

\section{Electron Microscopy}

For binding orientation experiments, VACV was bound to HeLa cells for 15 min on ice at MOI 50. Unbound virus was removed, and samples fixed for 20 min using (final concentration: 1.5\% 
formaldehyde, 0.1\%glutaraldehyde, both EM grade). Fix was replaced with 2\% glutaraldehyde in $0.1 \mathrm{M}$ sodium cacodylate for a further $20 \mathrm{~min}$. Samples were washed in $0.1 \mathrm{M}$ sodium cacodylate, incubated $1 \mathrm{~h}$ at $4^{\circ} \mathrm{C}$ in reduced osmium tetroxide. After additional washes, samples were dehydrated through an increasing ethanol series and critically point dried (Leica Auto CPD). Samples were Au/Pd coated and imaged using a FEI Quanta 200 FEG ESEM (Thermo Fisher Scientific) operated at $5 \mathrm{kV}$.

For fusion orientation experiments VACV was bound to HeLa cells for $30 \mathrm{~min}$ on ice at MOI

100. Unbound virus was removed, and samples incubated for $7.5 \mathrm{~min}$ at $37^{\circ} \mathrm{C}$ in DMEM with $100 \mathrm{mM}$ MES adjusted to $\mathrm{pH}$ 5. Samples were fixed with $1.5 \%$ glutaraldehyde $2 \%$ paraformaldehyde (EM-grade) in $0.1 \mathrm{M}$ sodium cacodylate for $45 \mathrm{~min}$ at $\mathrm{RT}$ and prepared for transmission electron microscopy (TEM). Transmission electron micrographs were obtained on a Tecnai T12 FEI equipped with a charge-coupled device camera (SIS Morada; Olympus).

\section{Code availability}

All custom code used for analysis in the current study is available on Github at https://github.com/HenriquesLab/fusiontools.

\section{Data availability:}

The datasets generated during and/or analysed during the current study are available from the corresponding author on reasonable request.

\section{References:}

1. Kielian, M. \& Rey, F. A. Virus membrane-fusion proteins: more than one way to make a hairpin. Nat. Rev. Microbiol. 4, 67-76 (2006).

2. Harrison, S. C. Viral membrane fusion. Nat. Struct. Mol. Biol. 15, 690-8 (2008). 
3. White, J. M., Delos, S. E., Brecher, M. \& Schornberg, K. Structures and mechanisms of viral membrane fusion proteins: multiple variations on a common theme. Crit. Rev. Biochem. Mol. Biol. 43, 189-219 (2008).

4. Moss, B. Fields Virology Poxviridae: The Viruses and Their Replication - Bernard Moss. (2006).

5. Moss, B. Poxvirus cell entry: How many proteins does it take? Viruses 4, 688-707 (2012).

6. Hsiao, J. C., Chung, C. S. \& Chang, W. Vaccinia virus envelope D8L protein binds to cell surface chondroitin sulfate and mediates the adsorption of intracellular mature virions to cells. J. Virol. 73, 8750-61 (1999).

7. Lin, C.-L., Chung, C.-S., Heine, H. G. \& Chang, W. Vaccinia Virus Envelope H3L Protein Binds to Cell Surface Heparan Sulfate and Is Important for Intracellular Mature Virion Morphogenesis and Virus Infection In Vitro and In Vivo. J. Virol. 74, 3353-3365 (2000).

8. Chiu, W.-L., Lin, C.-L., Yang, M.-H., Tzou, D.-L. M. \& Chang, W. Vaccinia Virus 4c (A26L) Protein on Intracellular Mature Virus Binds to the Extracellular Cellular Matrix Laminin. J. Virol. 81, 2149-2157 (2007).

9. Chung, C. S., Hsiao, J. C., Chang, Y. S. \& Chang, W. A27L protein mediates vaccinia virus interaction with cell surface heparan sulfate. J. Virol. 72, 1577-1585 (1998).

10. Senkevich, T. G., Ward, B. M. \& Moss, B. Vaccinia virus entry into cells is dependent on a virion surface protein encoded by the A28L gene. J. Virol. 78, 2357-66 (2004).

11. Ulaeto, D., Grosenbach, D. \& Hruby, D. E. The vaccinia virus 4c and A-type inclusion proteins ae specific markers for the intracellular mature virus particle. J. Virol. 70, 3372-3377 (1996).

12. Law, M., Carter, G. C., Roberts, K. L., Hollinshead, M. \& Smith, G. L. Ligand-induced and nonfusogenic dissolution of a viral membrane. Proc. Natl. Acad. Sci. U. S. A. 103, 5989-94 (2006).

13. Schmidt, F. I., Bleck, C. K. E., Helenius, A. \& Mercer, J. Vaccinia extracellular virions enter cells by macropinocytosis and acid-activated membrane rupture. EMBO J. 30, 3647-3661 (2011).

14. Doms, R. W., Blumenthal, R. \& Moss, B. Fusion of intra-and extracellular forms of vaccinia virus with the cell membrane. J. Virol. 64, 4884-4892 (1990).

15. Townsley, A. C., Weisberg, A. S., Wagenaar, T. R. \& Moss, B. Vaccinia virus entry into cells via a low-pHdependent endosomal pathway. J. Virol. 80, 8899-8908 (2006).

16. Gustafsson, M. G. L. Surpassing the lateral resolution limit by a factor of two using structured illumination microscopy. J. Microsc. 198, 82-87 (2000).

17. Gray, R. D. M. et al. VirusMapper: open-source nanoscale mapping of viral architecture through superresolution microscopy. Sci. Rep. 6, 29132 (2016).

18. Gray, R. D. M., Mercer, J. \& Henriques, R. Open-source Single-particle Analysis for Super-resolution Microscopy with VirusMapper. J. Vis. Exp. e55471-e55471 (2017). doi:10.3791/55471

19. Unger, B. \& Traktman, P. Vaccinia virus morphogenesis: a13 phosphoprotein is required for assembly of mature virions. J. Virol. 78, 8885-901 (2004).

20. Senkevich, T. G., Ojeda, S., Townsley, A., Nelson, G. E. \& Moss, B. Poxvirus multiprotein entry-fusion complex. Proc. Natl. Acad. Sci. U. S. A. 102, 18572-7 (2005).

21. Mirzakhanyan, Y. \& Gershon, P. The Vaccinia virion: Filling the gap between atomic and ultrastructure. PLoS Pathog. 15, e1007508 (2019).

22. Rust, M. J., Bates, M. \& Zhuang, X. Sub-diffraction-limit imaging by stochastic optical reconstruction microscopy (STORM). Nat. Methods 3, 793-796 (2006).

23. Su, H.-P., Golden, J. W., Gittis, A. G., Hooper, J. W. \& Garboczi, D. N. Structural basis for the binding of the neutralizing antibody, 7D11, to the poxvirus L1 protein. Virology 368, 331-341 (2007). 
24. Levet, F. et al. SR-Tesseler: a method to segment and quantify localization-based super-resolution microscopy data. Nat. Methods 12, 1065-71 (2015).

25. Laliberte, J. P., Weisberg, A. S. \& Moss, B. The membrane fusion step of vaccinia virus entry is cooperatively mediated by multiple viral proteins and host cell components. PLoS Pathog. 7, (2011).

26. Rodriguez, J. F., Paez, E. \& Esteban, M. A 14,000-Mr envelope protein of vaccinia virus is involved in cell fusion and forms covalently linked trimers. J. Virol. 61, 395-404 (1987).

27. Kochan, G., Escors, D., González, J. M., Casasnovas, J. M. \& Esteban, M. Membrane cell fusion activity of the vaccinia virus A17-A27 protein complex. Cell. Microbiol. 10, 149-64 (2008).

28. Sanderson, C. M., Hollinshead, M. \& Smith, G. L. The vaccinia virus A27L protein is needed for the microtubule-dependent transport of intracellular mature virus particles. J. Gen. Virol. 81, 47-58 (2000).

29. Rodriguez, J. F. \& Smith, G. L. IPTG-dependent vaccinia virus: Identification of a virus protein enabling virion envelopment by golgi membrane and egress. Nucleic Acids Res. 18, 5347-5351 (1990).

30. Vázquez, M. I. \& Esteban, M. Identification of functional domains in the 14-kilodalton envelope protein (A27L) of vaccinia virus. J. Virol. 73, 9098-109 (1999).

31. Kiskowski, M. A., Hancock, J. F. \& Kenworthy, A. K. On the Use of Ripley's K-Function and Its Derivatives to Analyze Domain Size. Biophys. J. 97, 1095-1103 (2009).

32. Schmidt, F. I., Kuhn, P., Robinson, T., Mercer, J. \& Dittrich, P. S. Single-Virus Fusion Experiments Reveal Proton Influx into Vaccinia Virions and Hemifusion Lag Times. Biophys. J. 105, 420-431 (2013).

33. Zawada, K. E., Okamoto, K. \& Kasson, P. M. Influenza Hemifusion Phenotype Depends on Membrane Context: Differences in Cell-Cell and Virus-Cell Fusion. J. Mol. Biol. 430, 594-601 (2018).

34. Chojnacki, J. et al. Maturation-Dependent HIV-1 Surface Protein Redistribution Revealed by Fluorescence Nanoscopy. Science (80-. ). 338, 524-528 (2012).

35. Mercer, J. \& Helenius, A. Vaccinia Virus Uses Macropinocytosis and Apoptotic Mimicry to Enter Host Cells. Science (80-. ). 320, 531-535 (2008).

36. Rizopoulos, Z. et al. Vaccinia Virus Infection Requires Maturation of Macropinosomes. Traffic 16, 814-831 (2015).

37. Huang, C.-Y. et al. A Novel Cellular Protein, VPEF, Facilitates Vaccinia Virus Penetration into HeLa Cells through Fluid Phase Endocytosis. J. Virol. 82, 7988-7999 (2008).

38. Vanderplasschen, A., Hollinshead, M. \& Smith, G. L. Intracellular and extracellular vaccinia virions enter cells by different mechanisms. J. Gen. Virol. 79, 877-887 (1998).

39. Sandgren, K. J. et al. A Differential Role for Macropinocytosis in Mediating Entry of the Two Forms of Vaccinia Virus into Dendritic Cells. PLoS Pathog. 6, e1000866 (2010).

40. Dales, S. The uptake and development of vaccinia virus in strain L cells followed with labeled viral deoxyribonucleic acid. J. Cell Biol. 18, 51-72 (1963).

41. Schmidt, F. I., Bleck, C. K. E. \& Mercer, J. Poxvirus host cell entry. Curr. Opin. Virol. 2, 20-27 (2012).

42. Schmidt, F. I. et al. Vaccinia Virus Entry Is Followed by Core Activation and Proteasome-Mediated Release of the Immunomodulatory Effector VH1 from Lateral Bodies. Cell Rep. 4, 464-476 (2013).

43. da Fonseca, F. G., Wolffe, E. J., Weisberg, A. \& Moss, B. Effects of deletion or stringent repression of the H3L envelope gene on vaccinia virus replication. J. Virol. 74, 7518-28 (2000).

44. Senkevich, T. G., Ward, B. M. \& Moss, B. Vaccinia Virus Entry into Cells Is Dependent on a Virion Surface Protein Encoded by the A28L Gene. J. Virol. 78, 2357-2366 (2004).

45. Ojeda, S., Domi, A. \& Moss, B. Vaccinia Virus G9 Protein Is an Essential Component of the Poxvirus Entry-Fusion Complex. J. Virol. 80, 9822-9830 (2006). 
46. Satheshkumar, P. S. \& Moss, B. Characterization of a newly identified 35-amino-acid component of the vaccinia virus entry/fusion complex conserved in all chordopoxviruses. J. Virol. 83, 12822-32 (2009).

47. Aldaz-Carroll, L. et al. Epitope-mapping studies define two major neutralization sites on the vaccinia virus extracellular enveloped virus glycoprotein B5R. J Virol 79, 6260-6271 (2005).

48. Szymborska, A. et al. Nuclear pore scaffold structure analyzed by super-resolution microscopy and particle averaging. Science (80-. ). 341, 655-658 (2013).

49. Heilemann, M. et al. Subdiffraction-resolution fluorescence imaging with conventional fluorescent probes. Angew. Chem. Int. Ed. Engl. 47, 6172-6 (2008).

50. Nahidiazar, L., Agronskaia, A. V., Broertjes, J., Van Broek, B. Den \& Jalink, K. Optimizing imaging conditions for demanding multi-color super resolution localization microscopy. PLoS One 11, 1-18 (2016).

51. Schindelin, J. et al. Fiji: an open-source platform for biological-image analysis. Nat. Methods 9, 676-682 (2012).

52. Ovesný, M., Křrižek, P., Borkovec, J., Švindrych, Z. \& Hagen, G. M. ThunderSTORM: A comprehensive ImageJ plug-in for PALM and STORM data analysis and super-resolution imaging. Bioinformatics 30, 2389-2390 (2014).

53. Laine, R. F. et al. NanoJ: a high-performance open-source super-resolution microscopy toolbox. J. Phys. D. Appl. Phys. 52, 163001 (2019).

54. White, J. Cell fusion by Semliki Forest, influenza, and vesicular stomatitis viruses. J. Cell Biol. 89, 674-679 (1981). 
541 We would like to thank Bernard Moss and Mariano Esteban for kindly providing mutant viruses 542 used in this study. This work was funded by MRC Programme Grant (MC_UU_00012/7) (J.M.), 543 the European Research Council (649101-UbiProPox) (J.M.), the UK Medical Research Council 544 (MR/K015826/1) (J.M., R.H.), Biotechnology and Biological Sciences Research Council $545 \quad(\mathrm{BB} / \mathrm{M} 022374 / 1 ; \quad \mathrm{BB} / \mathrm{P} 027431 / 1 ; \quad \mathrm{BB} / \mathrm{R} 000697 / 1) \quad(\mathrm{R} . \mathrm{H}),$.$\quad and the Wellcome Trust$ 546 (203276/Z/16/Z) (R.H.). R.G. is funded by the Engineering and Physical Sciences Research 547 Council (EP/M506448/1). D.A. is a Marie Sklodowska-Curie fellow funded by the European 548 Union (750673). C.B is funded by the Medical Research Council LMCB PhD program. We 549 thank Mark Turmaine, UCL Biosciences EM facility, and Andrew Weston, UCL School of 550 Pharmacy - Electron Microscopy Unit for use of their coater and SEM, respectively.

Author contributions: manuscript. R.G., D.A., C.B., and M.H. performed the experiments. The Cohen (G.H.C.) and Eisenberg members of the poxvirus research group produced and purified all VACV EFC antibodies. I.J.W. and J.J.B. performed electron microscopy. R.G., D.A. R.H and J.M analysed the data. R.G., D.A., R.H. and J.M. discussed the results and implications of the findings. All authors discussed and provided comments on the manuscript.

\section{Competing interests:}

Authors declare no competing interests.

\section{Materials and correspondence:}

Correspondence and requests for materials should be addressed to R.H. (r.henriques@ucl.ac.uk) or J.M. (jason.mercer@ucl.ac.uk). 


\section{Figure legends}

\section{Fig. 1. VACV binding and fusion show orientation bias reflecting distinct binding and}

fusion domains on virions. a, SEM images of VACV MV binding to the PM of HeLa cells. Quantification of binding orientation from $>50$ SEM images. b, TEM images of low $\mathrm{pH}$-induced fusion between VACV MVs and the PM of HeLa cells. Arrows indicate regions of continuity between virus and cell membranes. Quantification of fusion orientation from $>200$ cell sections. c, Localization models of binding and fusion proteins on MVs. d, Quantification of the polarity factor of proteins visualized in (c). A polarity factor $>1$ corresponds to concentration at the tips of MVs; $<1$ corresponds to concentration at the sides of MVs. e, Localization models of D8 and L1 in EFC and binding mutant MVs. f, Polarity factors for the models shown in (e) compared to WT. g, Illustration of VACV membrane protein organisation in $\mathrm{WT} / \Delta \mathrm{H} 3$ and fusion mutant MVs. Data represent 3 or more biological replicates (a-f). Models represent n>260 virions (c,e). Scale bars, 200nm (a-c,e). Bars represent means \pm SEM of $n=50$ virions (d,f).

\section{Fig. 2. VACV binding and fusion proteins are organized into nanoscale clusters.} a, Distribution of D8 (binding protein; first row) and L1 (fusion protein; second row) on individual virions imaged by STORM in WT, EFC mutant [A28(-), G9(-), O3(-)], and binding protein mutant $(\Delta \mathrm{H} 3) \mathrm{MVs}$. b, Voronoi diagrams and cluster identification of D8 and L1 in WT, EFC mutant, and binding mutants in (a). Voronoi tessellation was performed on individual virions with SR-Tesseler software (first row) and clusters identified with a density factor $\delta=3$ (second row). c, Percentage of D8 localizations within clusters on individual WT and mutant MVs. d, Number of D8 clusters identified per virion. e, Percentage of L1 localizations within 
clusters on individual WT and mutant MVs. f, Number of L1 clusters identified per virion. Images are representative of 3 biological replicates (a,b). Scale bars, 200nm (a,b). Data represent 3 or more biological replicates (c-f). Bars represent means \pm SEM of $n=15$ virions (c-f). An unpaired two-tailed $t$ test was applied $(* * * \mathrm{P}<0.001$, ns $\mathrm{P}>0.05$ ). See supplementary table 2 for exact statistics.

Fig. 3. A27 regulates the protein architecture of MV membranes. a, 24h yields from BSC40 cells infected with either A27(+) or A27(-) virus. b, Confocal images of A27(+) or A27(-) MV fusion from without experiments ( $\mathrm{pH}$ 5.0, MOI 50). Cell nuclei (magenta) and actin (green). c, Fusion index calculated for fusion from without experiments in (b) d, STORM images of A27 on individual MVs. e, VirusMapper models of D8 and L1 localization on A27(+) and A27(-) MVs. f, Polarity factor of models in (e). g, STORM images of D8 and L1 on A27(+) and A27(-) MVs. h, Percent of D8 and L1 clustered localizations on A27(+) and A27(-) MVs. i, Illustration of VACV membrane protein organization in WT/A27(+) vs. A27(-) MVs. Data represent 3 or more biological replicates (a,c,f,h). Images are representative of 3 biological replicates (b,d,g). Models represent $n>206$ virions (e). Bars represent means \pm SEM of $n=3$ replicates $(\mathbf{a}, \mathbf{c})$ or $n=50(\mathbf{f})$ or 15 (h) virions. Scale bars, $50 \mu \mathrm{m}(\mathbf{b})$ and $200 \mathrm{~nm}(\mathbf{d}, \mathbf{e , g})$.

\section{Fig. 4. VACV fusion machinery polarization is required for full fusion efficiency.} a, Schematic of VACV bulk fusion assays to quantify MV hemifusion by R18 dequenching, and full fusion by core EGFP quenching and recovery. b, Comparison of A27(+) and A27 (-) MV hemifusion rates by R18 dequenching assay. c, Comparison of A27(+) and A27 (-) MV full fusion rates using the EGFP core recovery assay. d, Analysis of the kinetics of A27 A27(+) and 
612 A27 (-) MV early gene expression by flow cytometry. e, Model of VACV density dependent 613 fusion kinetics. Virus-cell contact area was simulated with three different fusion complexes 614 densities (low, medium and high) using two different fusion thresholds (low and high). The 615 fusion rate (\% fused viruses/time) was modelled under these conditions. f, Model of VACV MV 616 and EEV protein architecture-dependent binding, entry and fusion. Data represent 3 biological 617 replicates $(\mathbf{b}-\mathbf{d})$. Lines represent mean \pm SD $(\mathbf{b}, \mathbf{c})$ or mean \pm SEM $(\mathbf{d})$ of $n=3$. Models were 618 generated using $\mathrm{n}=1000$ viruses/condition (e). 

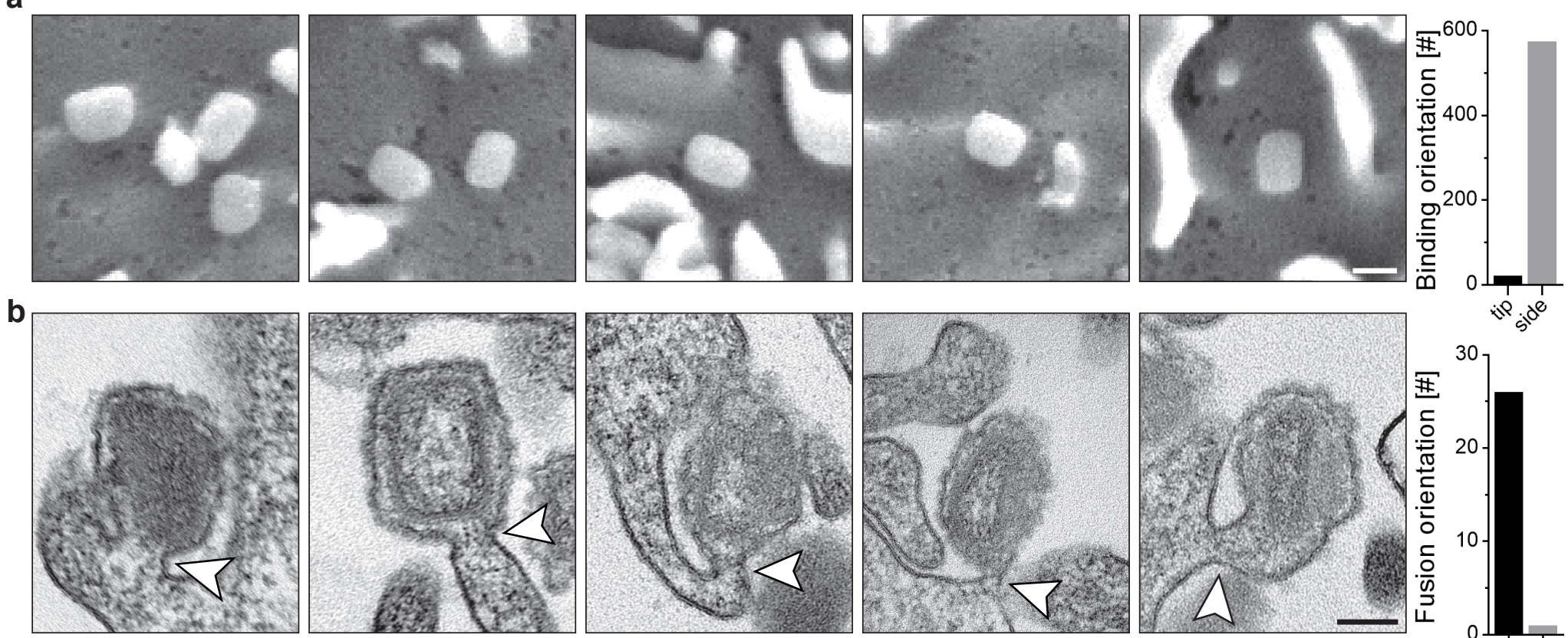

is $\dot{s}^{\circ}$

C Core

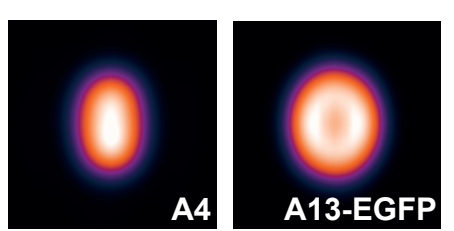

Binding
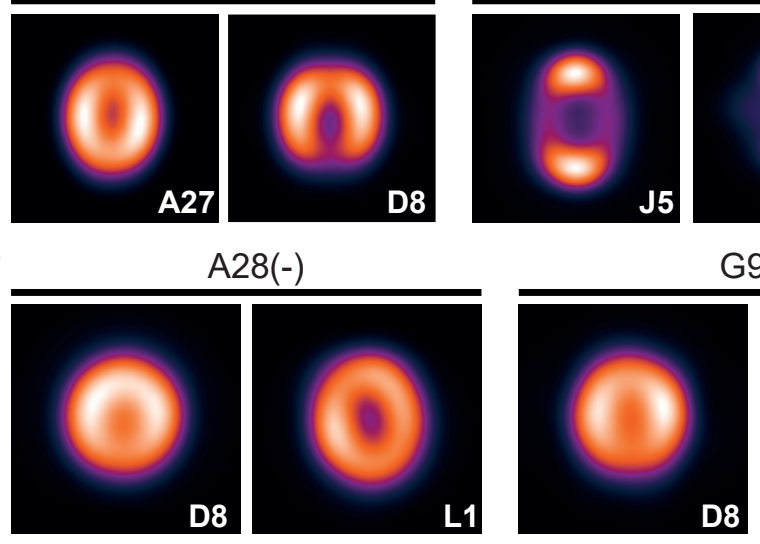

D8

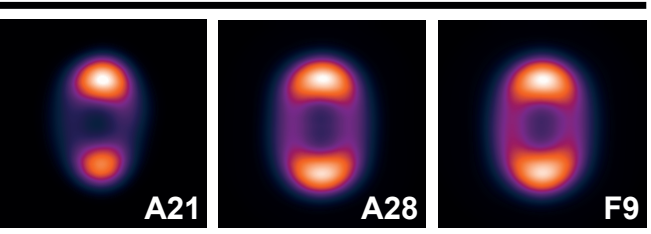

Fusion
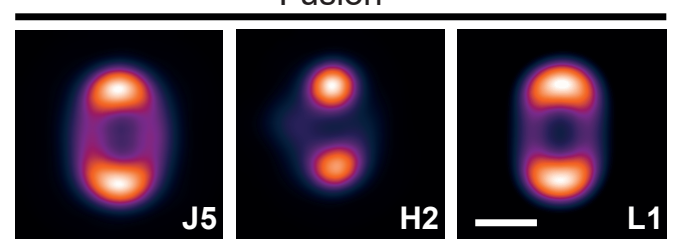

d
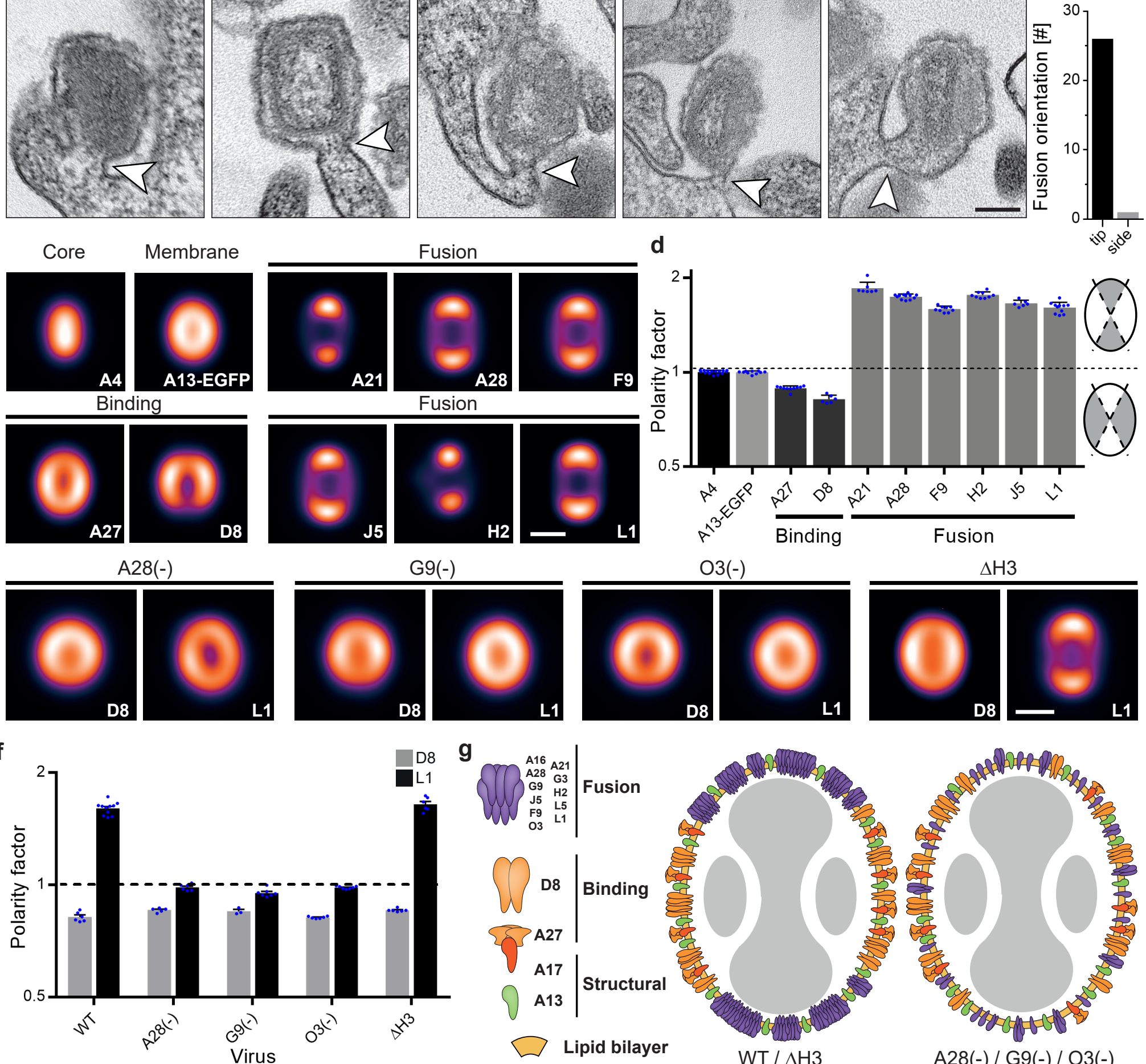

D8

L1
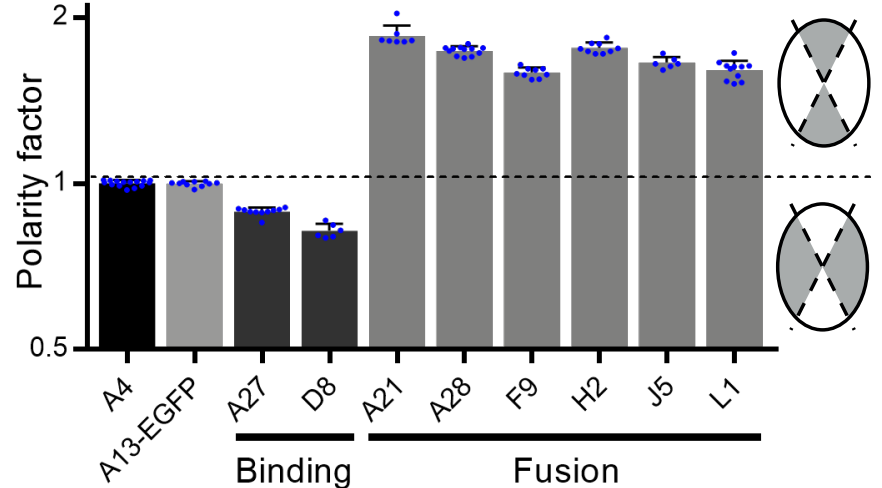

Fusion
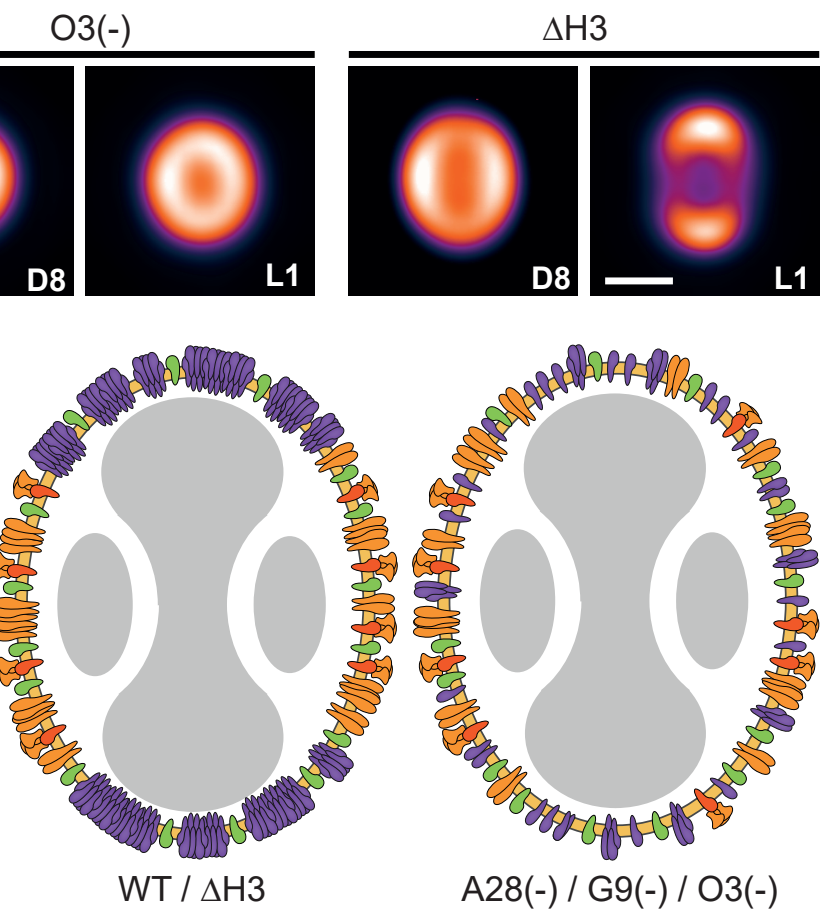
a

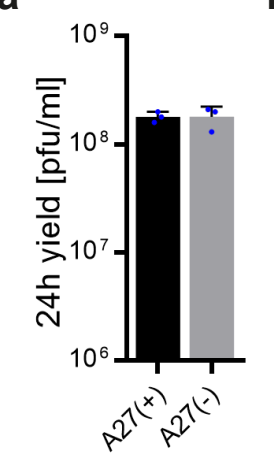

b
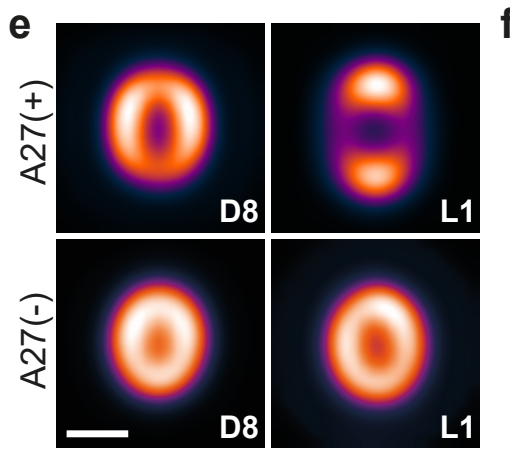

L1

i

\} \begin{tabular}{cc|l} 
A16 & A21 \\
G9 & G3 & Fusion \\
J5 & H2 & Fachinery \\
F9 & L1 \\
O3 & L1
\end{tabular} \mid macher

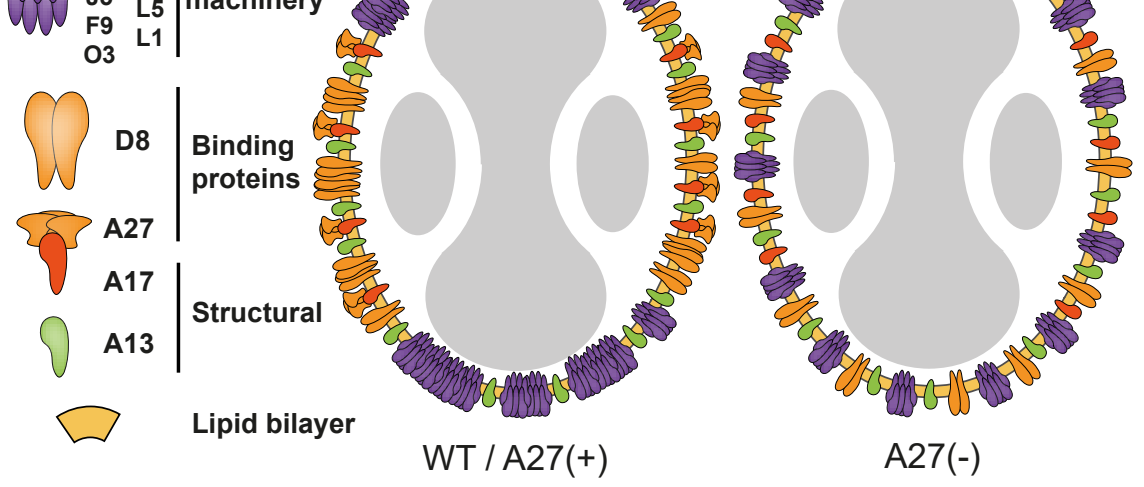

C
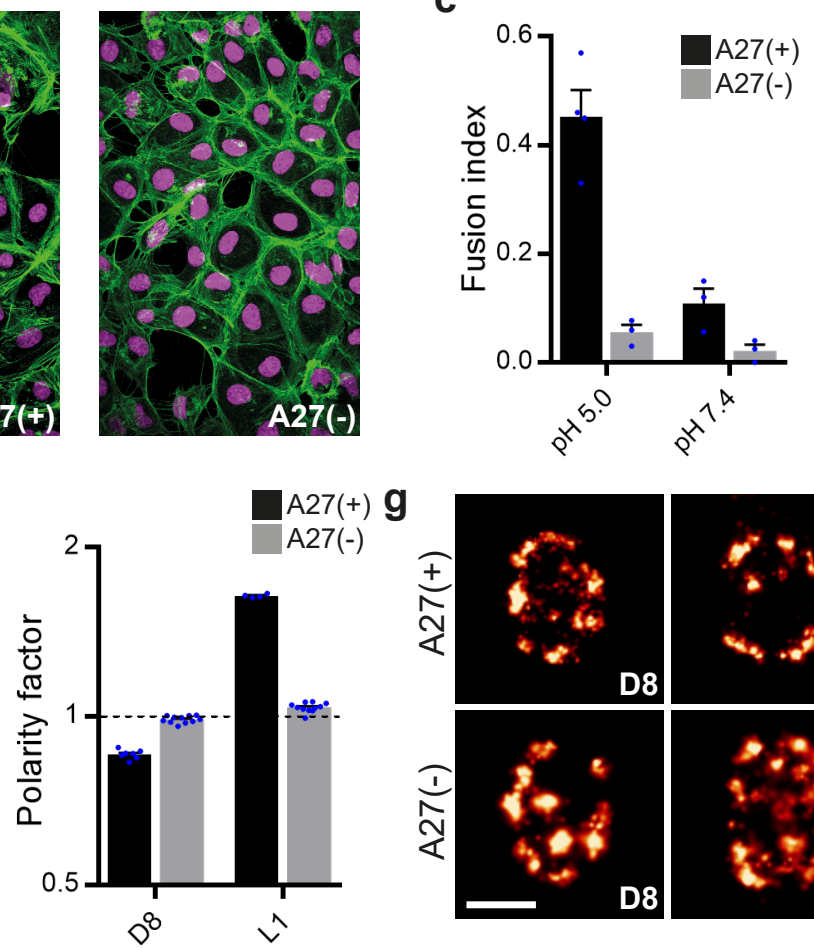

d
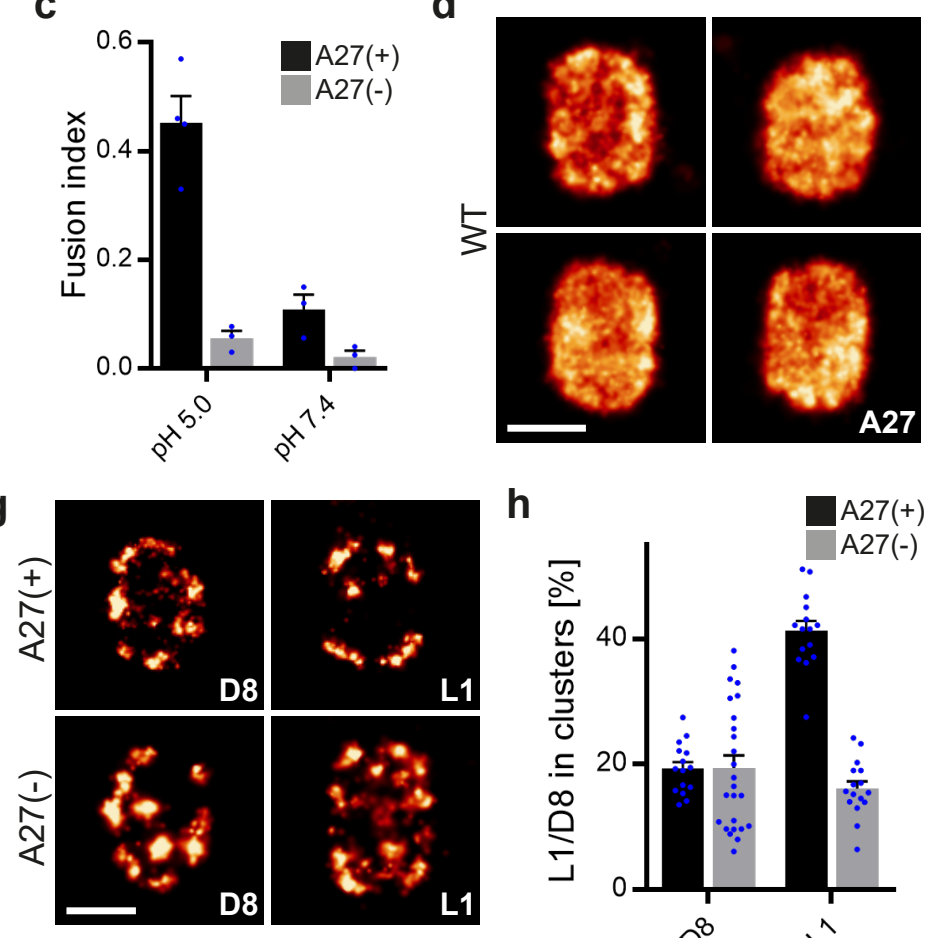

h

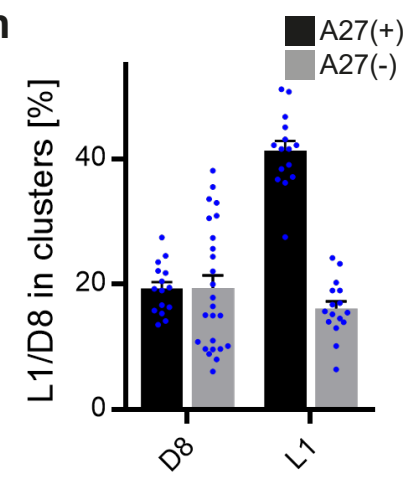


